Check for updates

Cite this: RSC Adv., 2017, 7, 39628

Received 2nd June 2017

Accepted 29th July 2017

DOI: $10.1039 / c 7 r a 06178 a$

rsc.li/rsc-advances

\title{
Polycaprolactone-templated reduced-graphene oxide liquid crystal nanofibers towards biomedical applications $\dagger$
}

\author{
Sasan Jalili-Firoozinezhad, (D) $\sharp \oint^{\text {ab }}$ Mohamad Hasan Mohamadzadeh Moghadam, $\dot{\dagger}^{\mathrm{c}}$ \\ Mohammad Hossein Ghanian, ${ }^{\mathrm{b}}$ Mohammad Kazemi Ashtiani, ${ }^{\mathrm{b}}$ Hossein Alimadadi, ${ }^{\mathrm{d}}$ \\ Hossein Baharvand, ${ }^{\text {*be }}$ Ivan Martin (D) ${ }^{* a}$ and Arnaud Scherberich ${ }^{a}$
}

\begin{abstract}
Here, we report a facile method to generate electrically conductive nanofibers by coating and subsequently chemically reducing graphene oxide (GO) liquid crystals on a polycaprolactone (PCL) mat. Ultra large GO sheets obtained are in favor of charge carrier mobility and oriented morphology of the GO coating. We showed that coating the reduced $\mathrm{GO}(\mathrm{rGO})$ not only retains the three-dimensional topography, fiber orientation and size of the template $\mathrm{PCL}$, but also makes it electroconductive. Our preliminary in vitro assessments using mesenchymal stem cells revealed no induced cytotoxicity yet increased cellular metabolism on PCL-templated rGO fibers.
\end{abstract}

\section{Introduction}

To date, electroactive nanofibrous substrates have been widely employed in various applications such as fuel cells, sensors and batteries. ${ }^{1}$ High surface-to-volume ratio, providing electrical cues and the capability to recapitulate the native extracellular matrix (ECM) architecture make electroconductive nanofibers an ideal platform for cell culture, tissue engineering, drug delivery and biosensor applications. ${ }^{2,3}$ Among different fabrication strategies, electrospinning serves as a facile, inexpensive, high-adaptable and scalable method to generate nanoscale fibrous structures from a wide variety of polymers. ${ }^{4-7}$

Graphene, a single layer carbon crystal with a packed honeycomb lattice structure, shows exceptional physicochemical, thermal and electrical properties as compared to other carbon materials. ${ }^{8-10}$ Thanks to its biocompatibility and potential to regulate cellular behaviors, graphene has been employed

\footnotetext{
${ }^{a}$ Department of Biomedicine, University Hospital Basel, University of Basel, Hebelstrasse 20, CH-4031 Basel, Switzerland. E-mail: ivan.martin@usb.ch

${ }^{b}$ Department of Stem Cells and Developmental Biology, Cell Science Research Center, Royan Institute for Stem Cell Biology and Technology, ACECR, Tehran, Iran. E-mail: baharvand@royaninstitute.org

${ }^{c}$ Condensed Matter National Laboratory, Institute for Research in Fundamental Sciences, Tehran, 19395-5531, Iran

${ }^{d}$ Center for Electron Nanoscopy, Technical University of Denmark, Fysikvej, Building 307, DK-2800 Kongens Lyngby, Denmark

${ }^{e}$ Department of Developmental Biology, University of Science and Culture, Tehran, Iran $\dagger$ Electronic supplementary information (ESI) available. See DOI: 10.1039/c7ra06178a

\$ These authors contributed equally.

$\S$ Current address: Wyss Institute for Biologically Inspired Engineering at Harvard University, Boston, MA 02115, USA. E-mail: sasan.jalili@wyss.harvard.edu.
}

in various biomedical fields. ${ }^{11,12}$ Although graphene can be formed in different shapes and architectures, efforts to make graphene nanofibers have been hampered by irregular shape and size of graphene sheets, and lack of efficient assembly methods. However, a new strategy has recently emerged to facilitate the generation of graphene oxide (GO) sheets and their coating on top of electrospun poly-L-lactide or poly(vinyl chloride) fiber meshes. ${ }^{13,14}$ Adapting this approach, we immobilized GO liquid crystal phases onto polycaprolactone (PCL) nanofibers, used as a template, and then reduced the GO (rGO), to form PCL-templated graphene nanofibers.

\section{Results and discussion}

In the current study, we took benefit of GO dispersion in the nematic phase, where large GO sheets could undergo liquid crystalline phase transition at very low concentration. While inter-sheet contacts and inter-layer gaps between small GO sheets compromise $2 \mathrm{D}$ conductivity, ${ }^{15}$ large GO liquid crystals obtained in the current work deliver excellent electrical properties. Having proper electrical conductivity and nanofibrous morphology at the same time would make our construct suitable for various biomedical applications.

GO was synthesized using the modified Hummers' method ${ }^{16}$ (Fig. 1A) in which, graphite oxide can be swelled in water (and polar organic compounds), ${ }^{17,18}$ followed by a spontaneous exfoliation as ions and impurities are being removed. ${ }^{17}$ This process was observed in our experiment as well (Fig. S1A $\dagger$ ). Chemical treatment of graphite introduces oxygenated functional groups on its surface and edges. Using Fourier transform infrared (FTIR) spectrum (Fig. 2A), the main functional groups were recognized: epoxy (1072 $\mathrm{cm}^{-1},-\mathrm{O}-$ stretching), hydroxyl 


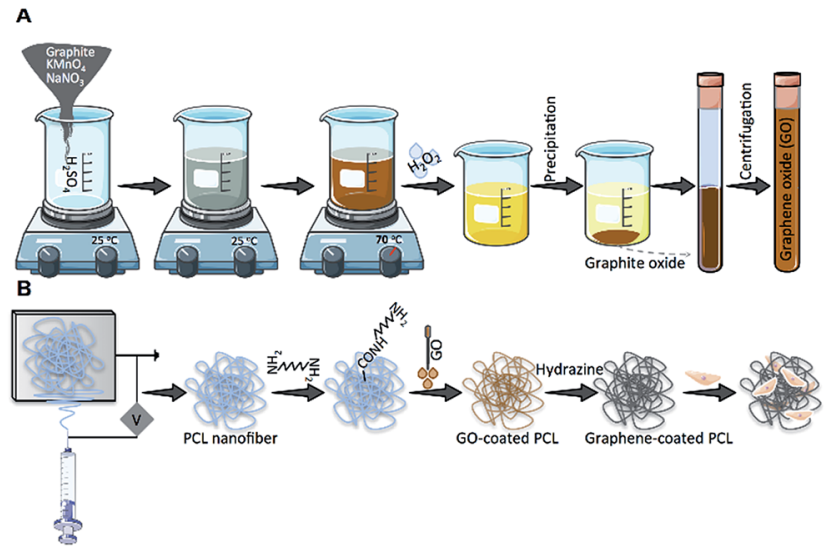

Fig. 1 Schematic illustration of (A) graphene oxide (GO) synthesis, (B) polycaprolactone $(\mathrm{PCL})$ electrospinning, fabrication of $\mathrm{rGO}$-coated $\mathrm{PCL}$ nanofibers (PCL-rGO) and in vitro cell seeding.
A
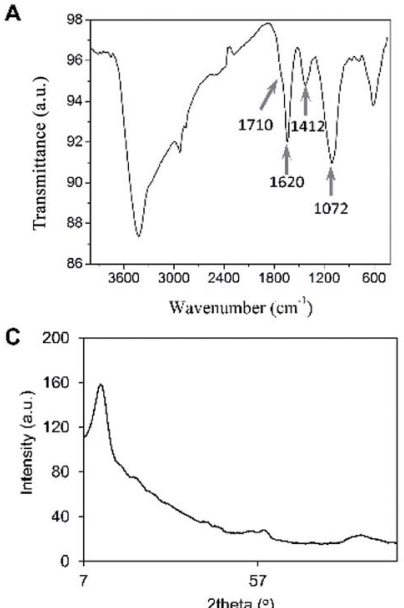

E

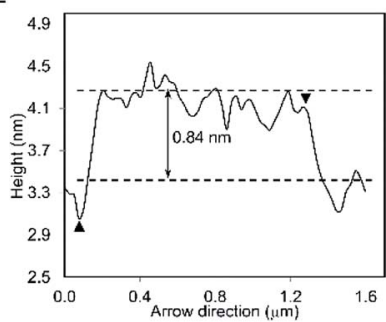

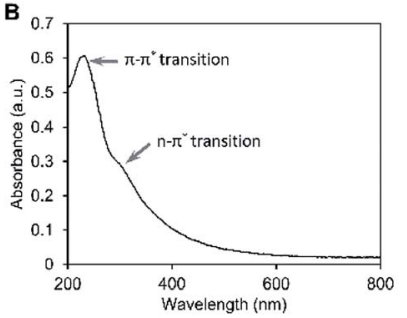

D

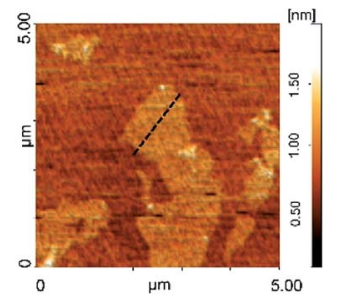

$\mathbf{F}$

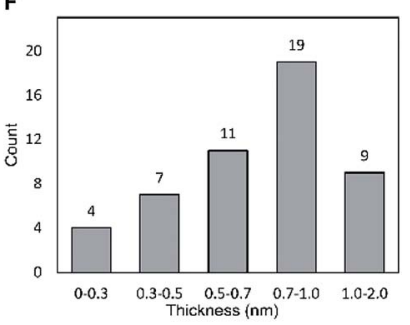

Fig. 2 Graphene oxide (GO) characterization. (A) FTIR spectrum of GO. The main peaks attributed to the functional groups on GO sheets were indicated by arrows. (B) UV-vis absorption spectrum of stable GO aqueous dispersion. (C) X-ray diffraction (XRD) pattern of GO film. (D) Representative atomic force microscopy (AFM) micrograph and (E) height profile of an exfoliated GO sheet (the cursor pair) showing single-layered structures with approximately $0.8 \mathrm{~nm}$ thickness. (F) Statistical analysis of sheet thickness by AFM performed on 50 randomly selected sheets.

(1412 $\mathrm{cm}^{-1}, \quad \mathrm{O}-\mathrm{H}$ deformation), carboxyl or carbonyl (1620 $\mathrm{cm}^{-1}$, O-H stretching and $1710 \mathrm{~cm}^{-1}, \mathrm{C}=\mathrm{O}$ stretching). ${ }^{19-21}$ Ultraviolet-visible (UV-vis) spectroscopy showed a distinct peak at $230 \mathrm{~nm}$ (due to $\pi-\pi *$ transition of $\mathrm{C}=\mathrm{C}$ bond in $\mathrm{sp}^{2}$-portion of GO) along with a shoulder at $300 \mathrm{~nm}$ (due to $\mathrm{n}-$ $\pi^{*}$ transition of $\mathrm{C}-\mathrm{O}$ bond in oxidized-portion of $\mathrm{GO}$ ) (Fig. 2B, S1B and $\mathrm{C} \dagger$ ).
The presence of oxygen moieties results in increment in interlayer distance between GO sheets. Based on XRD measurement, one peak centred at $11^{\circ}$ was found which is typical for GO sheets (Fig. 2C). By applying Bragg's law $(n \lambda=2 d \sin \theta)$, the $d$-spacing in GO was calculated around $0.8 \mathrm{~nm}$ which was in accordance with previous studies. ${ }^{18,22-24}$ The exfoliation of GO into single layer sheets has significant importance in the case of processing and eventual application. In this regard, atomic force microscopy (AFM) measurement was used to study the thickness of GO sheets. From images shown in Fig. $2 \mathrm{D}-\mathrm{F}$, a thickness of $\sim 0.8 \mathrm{~nm}$ was obtained which is in line with X-ray diffraction (XRD) result.

Electrical properties of GO coatings depend on its lateral size. ${ }^{25-27}$ Bigger lateral sizes of GO results in the higher electrical conductivity. ${ }^{28,29}$ Therefore, preserving lateral size of GO sheet during synthesis and fabrication is a key factor. Applying several centrifuging steps, small sheets were removed, hence, GO dispersion containing large sheets was obtained. Scanning electron microscopy (SEM) images of GO sheets alongside optical microscopy indicated the presence of large GO sheets (Fig. S2; 3A and C; S1D and E†). The size distribution of GO sheets was calculated as $21 \pm 12 \mu \mathrm{m}$ (Fig. 3B). Aqueous dispersion of large GO possesses a unique property in which it can undergo liquid crystalline phase transition at very low concentration. ${ }^{30,31}$ In other words, stacks of GO sheets in liquid phase will be oriented in a special direction which enables more facile fabrication process. ${ }^{30}$ As shown in Fig. 3D, GO dispersion is in the nematic phase at concentration of $0.25 \mathrm{mg} \mathrm{ml}^{-1}$. It
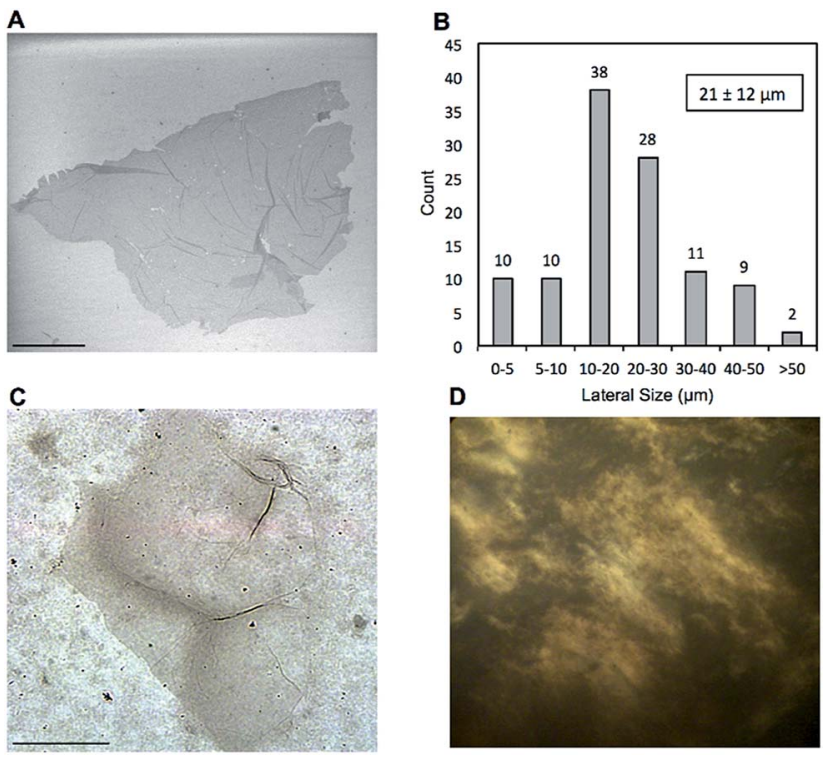

Fig. 3 (A) SEM image of large area of $\mathrm{GO}$ sheet on $\mathrm{Si} / \mathrm{SiO}_{2}$ substrate. Wrinkling can also be seen on basal plane of GO. Scale bar: $10 \mu \mathrm{m}$. (B) Size distribution of GO sheets calculated from 108 images. The average size is $21 \mu \mathrm{m}$ and the standard deviation is $12 \mu \mathrm{m}$. Minimum and maximum size was measured 2 and $51 \mu \mathrm{m}$, respectively. (C) Optical micrograph of large area GO sheet on coverslip. Scale bar: $20 \mu \mathrm{m}$. (D) Polarized optical microscopy (POM) micrograph of GO sheet at $0.25 \mathrm{mg} \mathrm{ml}^{-1}$. The bright part is due to orientation of stacks of $\mathrm{GO}$ sheet against incident light. 
should be taken into account that orientation of these sheets is favorable when achieving high electrical conductivity.

Electrospinning of graphene is quite challenging due to the irregular size/shape of chemically derived graphenes and the movable layer-by-layer stacking of graphenes, which could obstruct direct assembly of 2D microscopic graphene sheets into macroscopic nanofiber. Although many strategies have been devised to generate composite nanofiber substrates based on graphene,,$^{32,33}$ which fail to provide pure graphene interface, an alternative strategy is to use a polymeric nanofiber mat template, e.g. PCL, to immobilize GO on the surface (Fig. 1B). However, introduction of $-\mathrm{NH}_{2}$ groups onto the PCL mat template is essential for even and stable coating of GO sheets. The ninhydrin assay was used to determine the efficiency of hexamethylenediamine (HMD) in introducing amino groups on the surface of the PCL fibrous substrate. GO could be easily immobilized on surface through electrostatic attractions or hydrogen bonding between amino moieties of PCL on one side and hydroxyl, carboxyl and ether groups of GO on the other side. ${ }^{13,34} \mathrm{HMD}$, as an aminolyzing reagent, has been previously reported for inducing amino groups on PCL nanofibrous scaffolds for subsequent immobilization with biomolecules. HMD-aminolyzation enhances the cytocompatibility and hydrophilicity of PCL substrates without cross-reacting with, adsorbing to or changing diameter of PCL fibers. ${ }^{35,36}$ As described previously, ${ }^{35}$ UV-vis spectrophotometry was used to evaluate amination success. The presence of amino groups was confirmed by recording a more intense absorption band at $560 \mathrm{~nm}$ (32.5\% increase) on aminolyzed PCL (Fig. 4A). To obtain rGO-coated fibers, GO was first tightly immobilized onto clean PCL mat, which gave a brownish shade to the fibers' color. Through chemical reduction of GO-coated fibers by hydrazine vapor, rGO-coated substrates were obtained. Addition of hydrazine hydrate altered the GO color from brownish to dark gray, confirming the reduction of GO to rGO (Fig. 4B). To
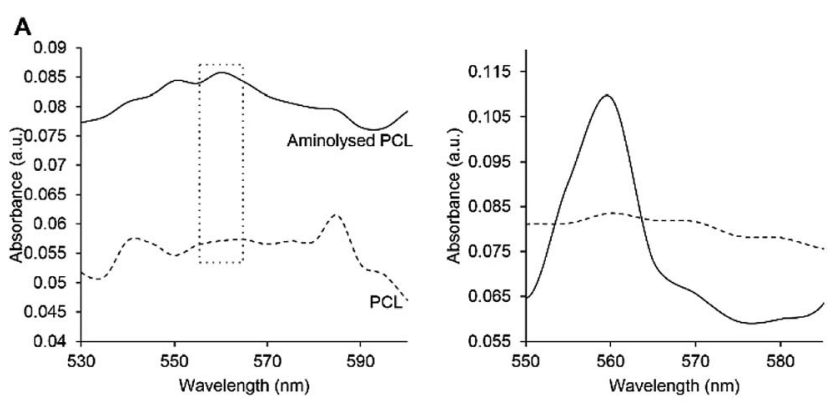

B

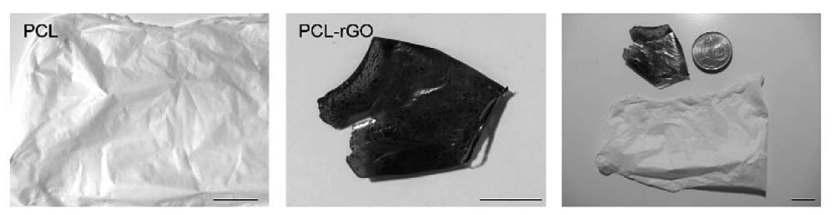

Fig. 4 (A) UV-vis spectra of polycaprolactone (PCL) and aminolysed $P C L$ samples. Right panel represents the difference between absorbance patterns of two substrates. (B) Digital images of electrospun polycaprolactone $(\mathrm{PCL})$ and graphene-coated $\mathrm{PCL}(\mathrm{PCL}-\mathrm{rGO})$ substrates. Scale bars: $10 \mathrm{~mm}$. evaluate morphological features, substrates were investigated by SEM. As shown in Fig. 5A, PCL nanofibers appeared to have a uniform randomly oriented bead-free morphology. As depicted in Fig. 5C-D, more than $50 \%$ of fibers were distributed in the size range of $400-1000 \mathrm{~nm}$, with average fiber diameter of $852.2 \pm 205.4 \mathrm{~nm}$. Graphene coating did not change the topography of PCL nanofibers (Fig. 5B), so that the average fiber diameter $(921.5 \pm 270.6 \mathrm{~nm})$ and distribution of PCL-rGO resembled those of PCL (Fig. 5C and D).

Fiber diameter plays an important role in modulating the adhesion, migration, proliferation and differentiation of cells. We used fibers with average diameter of 700-1000 $\mathrm{nm}$ which is known in neural engineering context to enhance elongation of neural stem cells and directing their differentiation towards neural lineages, compared to small $(200-400 \mathrm{~nm})$ and large $(1000-1500 \mathrm{~nm})$ dimensions. ${ }^{37,38}$ However, given the versatility of electrospinning to fabricate different sizes of PCL nanofiber platforms, our approach could be employed to fabricate rGOcoated nanofibers with various diameters to target specific biological responses. Although in previous studies fabrication of nanofiber structures based on GO has been reported, ${ }^{13,39}$ the above-described approach to employ GO liquid crystal phase has not been used so far to generate electro-conductive rGOcoated nanofibers. Comparing the in vitro behavior of stem cells on GO-versus rGO-based PCL fibers is beyond the scopes of this research; however, thanks to different degrees of $\pi-\pi$

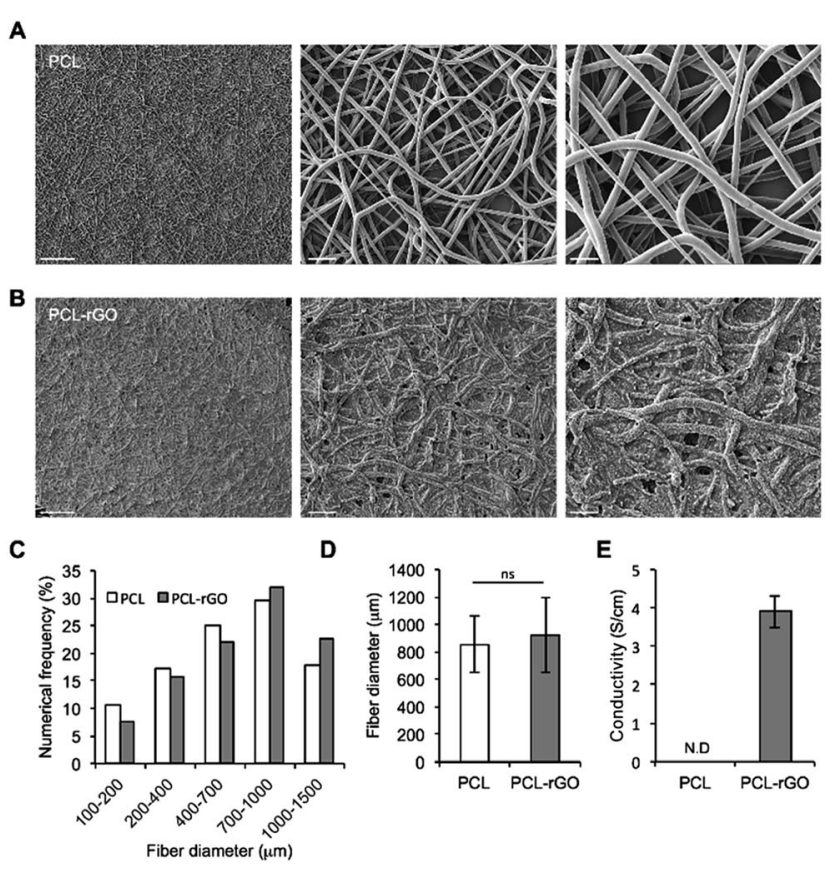

Fig. 5 Morphological and physical characterization of samples. (A) Representative field emission-scanning electron microscopy (FE-SEM) micrographs of (A) polycaprolactone $(\mathrm{PCL})$ nanofibers and $(\mathrm{B})$ graphene-coated PCL nanofibers (PCL-rGO). (C) Fiber diameter distribution and (D) average fiber diameter in intact and graphene coated nanofibers. (E) Electrical conductivity measurements of PCL and PCLrGO. Scale bar A - left panel: $100 \mu \mathrm{m}, \mathrm{B}$ - left panel: $50 \mu \mathrm{m}, \mathrm{A}$ - middle panel, B - middle panel: $30 \mu \mathrm{m}, \mathrm{A}$ - right panel, B - right panel: $5 \mu \mathrm{m}$. ns: not significant. 
stacking, hydrogen and electrostatic bonding, rGO has been shown to accelerate the differentiation of mesenchymal stromal/stem cells (MSCs) towards the osteogenic lineages ${ }^{12}$ or favor the maintenance of stem cells pluripotency ${ }^{\mathbf{4 0}}$ as compared to GO or inert substrates.

As illustrated in Fig. 5E, a four-point probe conductivity assay revealed that introduction of rGO onto the neat PCL fibers, resulted in induction of conductivity up to $3.9 \pm 0.4 \mathrm{~S} \mathrm{~cm}^{-1}$ in PCL-rGO. The relatively high electrical conductivity obtained in this work could be explained by the harnessing of two unique features of synthesized GO. First, the presence of ultra large GO sheets ensures the presence of conductive pathways within the coated layer, providing less physical junctions between neighboring sheets. ${ }^{41}$ Second, oriented GO sheets, adhered on the PCL substrate, are in favor of charge carrier mobility. Size of GO sheets affects their orientation on the PCL template. At very dilute GO dispersion with small lateral size, the isotropic phase, in which no orientation is achievable, is expected to be dominant $\left(0.1 \mathrm{mg} \mathrm{ml} \mathrm{m}^{-1}\right.$, Fig. 6A). This trend is observed even at a relatively higher concentration $\left(1 \mathrm{mg} \mathrm{ml}^{-1}\right.$, Fig. 6B). However, ultra large sheets enable nematic liquid crystalline phase to be dominant even at very dilute solutions $\left(0.1-0.2 \mathrm{mg} \mathrm{ml}^{-1}\right)$ used for dip coating process (Fig. 6C and D). This, in turn, favors adhesion of ordered GO sheet onto the PCL substrate, which is schematically shown in Fig. 7. Given the high electrical conductivity of PCL-rGO, this substrate could be harnessed for certain biological applications as compared to PCL per se. Electrically conductive nanofibers have been previously shown to increase cell attachment, sustain cell recovery and enhance neuronal/cardiomyogenic differentiation with or without external electrical stimulations as compared to nonconductive fibers. ${ }^{\mathbf{4 2}}$
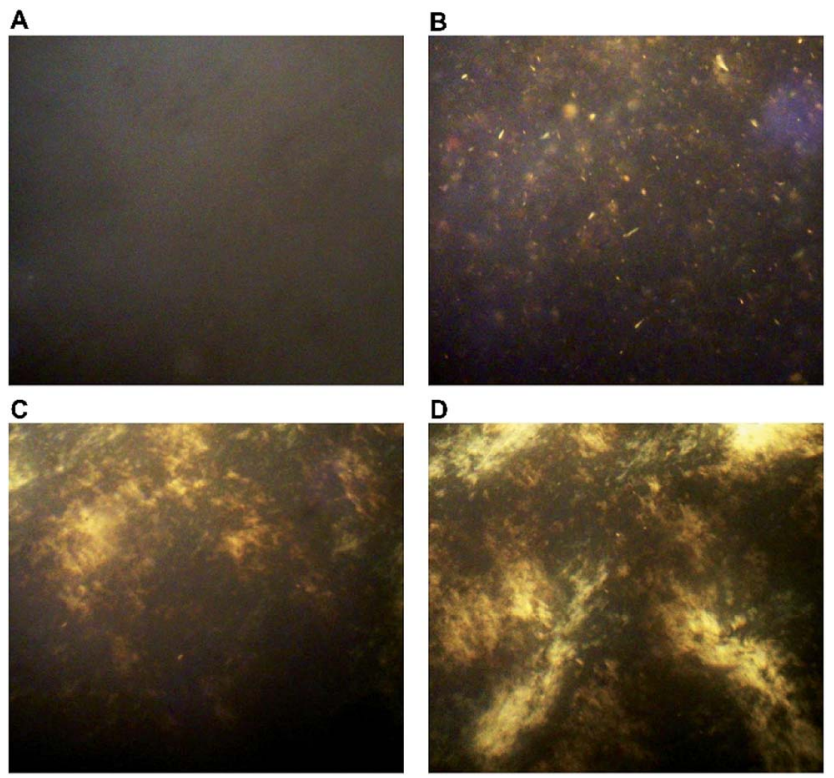

Fig. 6 Polarized optical microscopy (POM) images of aqueous GO dispersion with small lateral size $(0.6 \pm 0.5 \mu \mathrm{m})$ at concentration of $(\mathrm{A})$ $0.1 \mathrm{mg} \mathrm{ml}^{-1}$, and (B) $1 \mathrm{mg} \mathrm{ml}^{-1}$. POM images of aqueous $\mathrm{GO}$ dispersion with large lateral size $(21 \pm 12 \mu \mathrm{m})$ at concentration of (C) $0.1 \mathrm{mg} \mathrm{ml}^{-1}$, and (D) $0.2 \mathrm{mg} \mathrm{ml}^{-1}$.

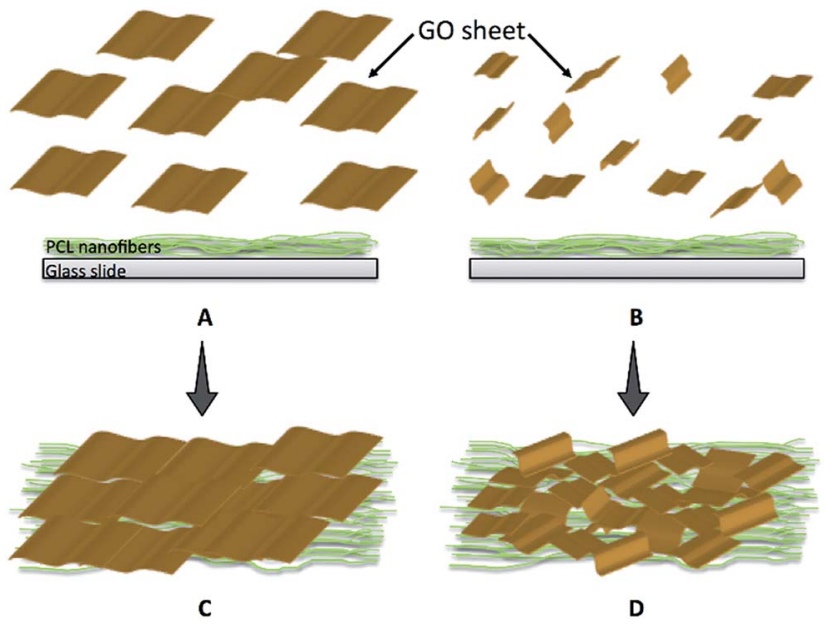

Fig. 7 Schematic representation of orientation of GO sheets on the PCL substrate: (A) nematic liquid crystalline phase containing large GO sheets in dilute solution which is in favor of oriented morphology of coating, and (B) isotropic phase of GO sheets in dilute solution when small GO sheets are used. The latter will result in disoriented film morphology in which GO sheets are randomly distributed in the coating. Schemes (C) and (D) depict the resultant films coated on the $P C L$ substrate from $(A)$ and $(B)$, respectively.

As initial steps to check whether introduction of rGO could affect the cytotoxicity of PCL fibers, analyses of viability and metabolic activity of MSCs on PCL and PCL-rGO substrates were performed at days 3, 7, and 14 using the MTS assay. Cell viability assessment confirmed that both samples were cytocompatible, with more than $80 \%$ viability in all culture intervals (Fig. 8A). However, as depicted in Fig. 8B, PCL-rGO showed significant increase $(P<0.05)$ in absorbance at $490 \mathrm{~nm}$ from day 7 to 14 of culture. Moreover, the absorbance value of PCL-rGO at day 14 was considerably higher $(P<0.05)$ than other samples, suggesting greater levels of cell metabolic activity and proliferation following rGO deposition. The extent of cellular metabolism and proliferation of MSCs have been previously reported to improve on graphene and other electro-conductive scaffolds in vitro. ${ }^{42-44}$

Overall, we aimed to develop a graphene-based nanofibrous substrate via facile approach, i.e. using the commonly used
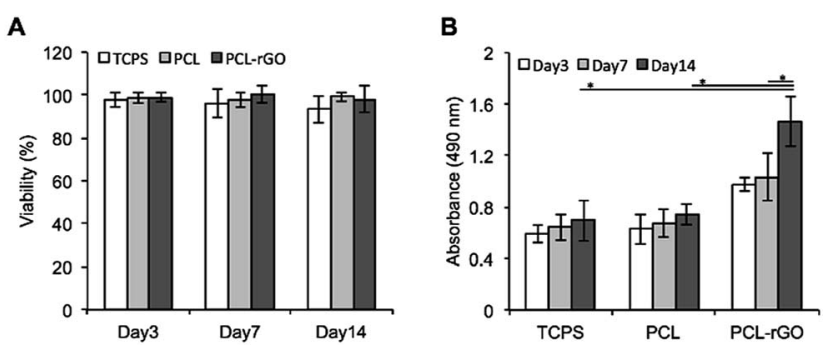

Fig. 8 In vitro biocompatibility of samples. MTS assay of mesenchymal stem cells (MSCs) at different intervals showing (A) viability and (B) metabolic activity of PCL and PCL-rGO substrates. Both samples are biocompatible, while the metabolism of cells on PCL-rGO at day 14 is higher than PCL and standard tissue culture polystyrene (TCPS) samples. $(n=4, * P<0.05, * * P<0.01)$. 
electrospun PCL template. Thorough in vitro investigations were beyond the prospects of the current work; however, having the fibrous and electro-conductive features, combined, this construct could be later employed to study the differentiation behavior of MSCs in absence or presence of external electrical stimulations. Electroconductive nanofibers show great promise in tissue engineering area, since they provide ECM-like topographical cues and deliver electrical signals to cells simultaneously and thus, promote electroactive tissue repair. ${ }^{45}$

\section{Conclusions}

In this study, we fabricated electroconductive rGO nanofibers via a simple process by immobilization of GO liquid crystals on electrospun PCL nanofibers. We used PCL nanofibers as a template to coat and reduce GO liquid crystals. Ultra large GO liquid crystals obtained in the current study are in favour of charge carrier mobility and affect the orientation of GO sheets on the PCL template. Moreover, the large GO liquid crystals enable conductive pathways within the coated layers and reduce physical junctions between adjacent sheets, thus providing high electrical conductivity in the resulting PCL-templated rGO fibers. Preliminary in vitro analyses confirmed that rGO nanofibers support active metabolism of human MSCs with enhanced cellular proliferation as compared to standard cell culture plates and PCL nanofibers. Our future studies will aim to assess functional properties of the PCL-rGO towards regeneration of electro-responsive tissues such as muscle and myocardium.

\section{Experimental}

\section{Graphene oxide (GO) synthesis}

To generate GO aqueous dispersion, graphite flakes (1 g, Merck) were added to $\mathrm{H}_{2} \mathrm{SO}_{4}$ solution ( $80 \mathrm{ml}, 98 \% \mathrm{w} / \mathrm{v}$, Merck) and remained under constant stirring for $2 \mathrm{~h}$. Following the addition of $\mathrm{KMnO}_{4}(4-6 \mathrm{~g}$, Sigma) to the mixture and stirring for an overnight, the resultant solution first turned to dark green during the oxidation process of graphite, and then changed to dark brown. Next, double distilled water (d.d. $\mathrm{H}_{2} \mathrm{O}, 300 \mathrm{ml}$ ) was added to quench the oxidation reaction. To inactivate residual (un/non-reacted) $\mathrm{KMnO}_{4}$, aqueous solution of $\mathrm{H}_{2} \mathrm{O}_{2}(10 \mathrm{ml}, 10 \%$ $\mathrm{w} / \mathrm{v}$, Merck) was added, which gave yellowish color to the mixture (Fig. S1A $\dagger$ ). To remove excess ions and acids from the suspension, the mixture was centrifuged at least three times with d.d. $\mathrm{H}_{2} \mathrm{O}$ and $\mathrm{HCl}(1 \mathrm{M}$, Merck) at $8000 \mathrm{rpm}$. GO formed after spontaneous exfoliation process in graphite oxide. Finally, to remove unexfoliated particles, GO dispersion was purified through dialysis (12 kDa tube) process in d.d. $\mathrm{H}_{2} \mathrm{O}$ for $72 \mathrm{~h}$ at $25{ }^{\circ} \mathrm{C}$. Schematic representation of GO synthesis was depicted in Fig. 1A.

\section{Fabrication of polycaprolactone (PCL) nanofibers}

To generate electrospun nanofibrous substrate, a polymer solution with overall concentration of $12 \mathrm{wt} \%$ was obtained by dissolving PCL (80 kDa, Sigma) in chloroform : methanol (3:1 $\mathrm{v} / \mathrm{v}$, Merck). The solution was electrospun via a $10 \mathrm{ml}$ syringe with a 20 gauge blunt needle at a flow rate of $1 \mathrm{ml} \mathrm{h}^{-1}$. After applying a high voltage to the needle $(14 \mathrm{kV})$, nanofibers were collected on a grounded drum placed at a distance of $17 \mathrm{~cm}$ from the needle tip and rotating at $250 \mathrm{rpm}$. Electrospun fibers were then vacuum dried at $25^{\circ} \mathrm{C}$ for $24 \mathrm{~h}$ to remove any residual solvent remained in the construct and kept in a desiccator for further investigations.

\section{Surface modification of PCL electrospun fibers mat}

Prior to perform aminolysis of PCL fibrous substrates, they were rinsed in ethanolic aqueous solution $(50 \% \mathrm{v} / \mathrm{v})$ for $2-3 \mathrm{~h}$ followed by several washes in d.d. $\mathrm{H}_{2} \mathrm{O}$. Aminolysis procedure was carried out by immersing the fiber mats in a hexamethylenediamine (HMD)/2-propanol (Sigma) solution (12\% w/v) for $24 \mathrm{~h}$ at $37{ }^{\circ} \mathrm{C}$ in a shaker incubator. The aminolyzed fibers were then rinsed several times with d.d. $\mathrm{H}_{2} \mathrm{O}$ for $24 \mathrm{~h}$ at $25{ }^{\circ} \mathrm{C}$ and subsequently vacuum dried at $30{ }^{\circ} \mathrm{C}$ for $24 \mathrm{~h}$ until of a constant weight.

\section{Graphene-coated on PCL nanofibers}

To cover the PCL nanofibers with reduced graphene (rGO), fiber mats were immersed into the GO solution $\left(1 \mathrm{mg} \mathrm{ml}^{-1}\right)$ for $2 \mathrm{~h}$. rGO-Coated fibers were obtained by keeping the samples under hydrazine monohydrate vapor (20\% aqueous solution, Merck) in a desiccator for $24 \mathrm{~h}$ at $40{ }^{\circ} \mathrm{C}$, after which the color of PCL fibers transformed from white to greyish black due to the reduction of GO. The samples were then vacuum dried for $24 \mathrm{~h}$ at $40{ }^{\circ} \mathrm{C}$ and rinsed several times for $24 \mathrm{~h}$ with d.d. $\mathrm{H}_{2} \mathrm{O}$ (Fig. 1B).

\section{Characterizations}

To analyze the topology profiles of exfoliated GO sheets, following the deposition of a GO solution on a freshly cleaved mica surface, atomic force microscopy (AFM) images were obtained by a Dual scope 95-200 (DME, Denmark) working under tapping mode. X-ray diffraction (XRD) pattern of GO was obtained using a Philips X-ray spectrometer (Netherlands) after drying the GO dispersion in a lyophilizer (Christ, Alpha 1-2 LD, Germany). Ultraviolet-visible (UV-vis) spectrum of GO was obtained using a Nicolet Evolution spectrophotometer (Thermo Scientific, USA) in the wavelength range of $200-800 \mathrm{~nm}$. To evaluate the heterogeneity and birefringence of GO dispersion polarized optical microscopy (POM) was performed by Leica DMR microscope in transmission mode. Optical images were recorded by placing a drop of dispersion on a glass coverslip and observed under an inverted microscope (BX51, Olympus) equipped with an Olympus DP72 digital camera. To characterize the size and size distribution of GO flakes, secondary electron (SE) imaging was applied in an FEI Helios NanoLab ${ }^{\mathrm{TM}}$ 600 dual beam scanning electron microscope. To ensure high quality imaging, GO flakes were dispersed on $\mathrm{SiO}_{2}$ substrate and imaged by electron probe current of $11 \mathrm{pA}$ at an acceleration voltage of $2 \mathrm{kV}$. To evaluate the morphology and architecture of GO, PCL and PCL-rGO substrates, samples were observed by a field emission scanning electron microscopy (FE-SEM, FEI Nova NanoSEM 230, USA). Average fiber diameter and 
distribution were determined using Image Analyzer software (Image J 1.44p). The electrical conductivity of PCL and PCL-rGO substrates was measured by a four-point probe method using Keithley instrument (Model 196 System DMM, USA). The resistivity of substrates with determined thickness $(t)$ was obtained using the following equation: $\rho=(\pi / \ln 2) \times(V / I) \times t$, where $V$ is the measured voltage and $I$ is the applied current. The conductivity of samples was obtained as the inverse of resistivity.

\section{Determination of the amino groups}

To detect the presence of $\mathrm{NH}_{2}$ groups on the aminolyzed PCL fiber mats, ninhydrin assay was performed. The substrates were first immersed in ninhydrin/ethanol solution (1 M, Sigma) for $1 \mathrm{~min}$ and then heated at $80{ }^{\circ} \mathrm{C}$ for $10 \mathrm{~min}$ to facilitate ninhydrin-amino reaction, which in turn made the surface of nanofibers blue. To dissolve the PCL mat, 1,4-dioxane (Merck) was added and to stabilize the blue compound, isopropanol (Merck) was introduced into the mixture. The UV-vis spectra of the modified and neat nanofibers were recorded in the range of 450-650 nm to determine the presence of $\mathrm{NH}_{2}$ groups on the surface of modified PLC nanofibers.

\section{In vitro studies}

Cell seeding onto substrates. Prior to cell seeding, samples were rinsed overnight with $70 \%$ ethanol, sterile phosphate buffered saline (PBS, Invitrogen) for $24 \mathrm{~h}$ and cell culture media overnight, followed by $2 \mathrm{~h} \mathrm{UV}$ radiation. Human bone marrow derived mesenchymal stromal/stem cells (MSCs) were isolated as described previously ${ }^{46}$ from bone marrow aspirates $(20 \mathrm{ml}$ volumes) of healthy donors $(n=5)$ obtained during orthopaedic surgical procedures after informed consent and in accordance with the local ethical committee (University Hospital Basel; approval date 26/03/2007 Ref. number 78/07). MSCs were incubated in alpha-MEM supplemented with $10 \%$ fetal bovine serum (FBS, Gibco), 1\% s-glutamine (Gibco) and 1\% penicillin/ streptomycin (Gibco) at $37{ }^{\circ} \mathrm{C}$ in a humidified incubator with $5 \% \mathrm{CO}_{2}$. At confluency, MSCs were trypsinized and seeded (105 cells per $\mathrm{cm}$ ) on substrates.

Cytotoxicity evaluation. To determine the biocompatibility of the PCL and PCL-rGO substrates, in vitro cytotoxicity analyses using a MTS assay were performed on the substrates' extracts and compared with standard control, tissue culture polystyrene (TCPS). Following the incubation of samples in culture media $\left(0.1 \mathrm{~cm}^{2} \mathrm{ml}^{-1}\right)$ at $37^{\circ} \mathrm{C}$, extracts were collected after 3,7 and 14 days. The MSCs were then incubated for $24 \mathrm{~h}$ in 96-well plates $\left(10^{4}\right.$ cells per well) in culture medium and subsequently exposed to the sample extract and incubated overnight at $37{ }^{\circ} \mathrm{C}$ and $5 \%$ $\mathrm{CO}_{2}$. After removing the extract medium, CellTiter $96 \AA$ Aqueous MTS reagent (Promega, G5421) was added to the cells and plates were placed at $37^{\circ} \mathrm{C}$ in the dark. The absorbance of the solution was measured at $490 \mathrm{~nm}$ and relative cell viability was obtained by having the absorbance of samples $\left(a_{\mathrm{s}}\right)$ and control group $\left(a_{\mathrm{c}}\right)$ as follows: viability $(\%)=\left(a_{\mathrm{s}} / a_{\mathrm{c}}\right) \times 100$. Three parallels were averaged for each sample.
Cell proliferation on substrates. Proliferation of MSCs on PCL and PCL-rGO samples was determined using MTS assay and according to the manufacturer's protocol. In short, after 3, 7 and 14 days, the cell seeded substrates were transferred into new wells and treated with MTS solution for $3 \mathrm{~h}$ in a $5 \% \mathrm{CO}_{2}$ incubator and without the presence of light. The absorbance was measured at $490 \mathrm{~nm}$ and the average of four readings was calculated for each sample.

\section{Statistical analysis}

Data were expressed as mean \pm standard deviation (SD). Statistical differenced in the measured properties between groups were analyzed by one-way analysis of variance (ANOVA) and the independent Student's $t$-test using SPSS 16.0 software. Post hoc analysis was performed using Tukey's HSD tests. $P$ values of less than 0.05 were considered significant.

\section{Conflicts of interest}

There are no conflicts to declare.

\section{Acknowledgements}

This work was supported by a grant provided by a Swiss excellence scholarship from the Swiss National Science Foundation to S. J.-F. (SNF grant \#2013.0022) and by a grant from Royan Institute to S. J.-F. and M. H. M. M. We are grateful to Ms Evi Bieler (Zentrum fur Mikroskopie, University of Basel) for her excellent technical assistance with scanning electron microscopy.

\section{Notes and references}

1 L. Persano, A. Camposeo and D. Pisignano, Prog. Polym. Sci, 2015, 43, 48-95.

2 S. Jalili-Firoozinezhad, F. Mirakhori and H. Baharvand, in Stem-Cell Nanoengineering, ed. H. Baharvand and N. Aghdami, John Wiley \& Sons, Inc, 2015, pp. 265-283.

3 G. Kaur, R. Adhikari, P. Cass, M. Bown and P. Gunatillake, RSC Adv., 2015, 5, 37553-37567.

4 S. Tavakol, S. Jalili-Firoozinezhad, O. Mashinchian and M. Mahmoudi, in Nanoscience in Dermatology, ed. M. R. Hamblin, P. Avci and T. W. Prow, Academic Press, Boston, 2016, pp. 337-352.

5 S. H. Lim and H.-Q. Mao, Adv. Drug Delivery Rev., 2009, 61, 1084-1096.

6 S. Agarwal, J. H. Wendorff and A. Greiner, Polymer, 2008, 49, 5603-5621.

7 Z. Su, J. Ding and G. Wei, RSC Adv., 2014, 4, 52598-52610.

8 A. Savchenko, Science, 2009, 323, 589-590.

9 Editorial article, Nat. Nanotechnol., 2008, 3, 517.

10 Y. Li, P. Zhang, Z. Ouyang, M. Zhang, Z. Lin, J. Li, Z. Su and G. Wei, Adv. Funct. Mater., 2016, 26, 2122-2134.

11 C. Chung, Y.-K. Kim, D. Shin, S.-R. Ryoo, B. H. Hong and D.-H. Min, Acc. Chem. Res., 2013, 46, 2211-2224. 
12 W. C. Lee, C. H. Y. X. Lim, H. Shi, L. A. L. Tang, Y. Wang, C. T. Lim and K. P. Loh, ACS Nano, 2011, 5, 7334-7341.

13 K. Zhang, H. Zheng, S. Liang and C. Gao, Acta Biomater., 2016, 37, 131-142.

14 Z.-Q. Feng, T. Wang, B. Zhao, J. Li and L. Jin, Adv. Mater., 2015, 27, 6462-6468.

15 X. Lin, J. Jia, N. Yousefi, X. Shen and J.-K. Kim, J. Mater. Chem. C, 2013, 1, 6869-6877.

16 S. H. Aboutalebi, M. M. Gudarzi, Q. B. Zheng and J.-K. Kim, Adv. Funct. Mater., 2011, 21, 2978-2988.

17 M. M. Gudarzi, M. H. M. Moghadam and F. Sharif, Carbon, 2013, 64, 403-415.

18 R. Jalili, S. H. Aboutalebi, D. Esrafilzadeh, K. Konstantinov, S. E. Moulton, J. M. Razal and G. G. Wallace, ACS Nano, 2013, 7, 3981-3990.

19 M. H. Mohamadzadeh Moghadam, S. Sabury, M. M. Gudarzi and F. Sharif, J. Polym. Sci., Part A: Polym. Chem., 2014, 52, 1545-1554.

20 C.-M. Chen, J.-Q. Huang, Q. Zhang, W.-Z. Gong, Q.-H. Yang, M.-Z. Wang and Y.-G. Yang, Carbon, 2012, 50, 659-667.

21 D. C. Marcano, D. V. Kosynkin, J. M. Berlin, A. Sinitskii, Z. Sun, A. Slesarev, L. B. Alemany, W. Lu and J. M. Tour, ACS Nano, 2010, 4, 4806-4814.

22 A. M. Dimiev, L. B. Alemany and J. M. Tour, ACS Nano, 2013, 7, 576-588.

23 T. Szabó, E. Tombácz, E. Illés and I. Dékány, Carbon, 2006, 44, 537-545.

24 A. Dimiev, D. V. Kosynkin, L. B. Alemany, P. Chaguine and J. M. Tour, J. Am. Chem. Soc., 2012, 134, 2815-2822.

25 X. Wang, H. Bai and G. Shi, J. Am. Chem. Soc., 2011, 133, 6338-6342.

26 J. Chen, Y. Li, L. Huang, N. Jia, C. Li and G. Shi, Adv. Mater., 2015, 27, 3654-3660.

27 X. Lin, X. Shen, Q. Zheng, N. Yousefi, L. Ye, Y.-W. Mai and J.-K. Kim, ACS Nano, 2012, 6, 10708-10719.

28 G. Eda, G. Fanchini and M. Chhowalla, Nat. Nanotechnol., 2008, 3, 270-274.

29 J. Zhao, S. Pei, W. Ren, L. Gao and H.-M. Cheng, ACS Nano, 2010, 4, 5245-5252.
30 S. Naficy, R. Jalili, S. H. Aboutalebi, R. A. G. Iii, K. Konstantinov, P. C. Innis, G. M. Spinks, P. Poulin and G. G. Wallace, Mater. Horiz., 2014, 1, 326-331.

31 R. Jalili, S. H. Aboutalebi, D. Esrafilzadeh, K. Konstantinov, J. M. Razal, S. E. Moulton and G. G. Wallace, Mater. Horiz., 2013, 1, 87-91.

32 W. Guo, X. Zhang, X. Yu, S. Wang, J. Qiu, W. Tang, L. Li, H. Liu and Z. L. Wang, ACS Nano, 2016, 10, 5086-5095.

33 C. Chen, T. Zhang, Q. Zhang, X. Chen, C. Zhu, Y. Xu, J. Yang, J. Liu and D. Sun, ACS Appl. Mater. Interfaces, 2016, 8, 1018310192.

34 Y. Zhu, C. Gao, X. Liu and J. Shen, Biomacromolecules, 2002, 3, 1312-1319.

35 W. Mattanavee, O. Suwantong, S. Puthong, T. Bunaprasert, V. P. Hoven and P. Supaphol, ACS Appl. Mater. Interfaces, 2009, 1, 1076-1085.

36 S. Regis, S. Youssefian, M. Jassal, M. D. Phaneuf, N. Rahbar and S. Bhowmick, J. Biomed. Mater. Res., Part A, 2014, 102, 1697-1706.

37 G. T. Christopherson, H. Song and H.-Q. Mao, Biomaterials, 2009, 30, 556-564.

38 H. B. Wang, M. E. Mullins, J. M. Cregg, C. W. McCarthy and R. J. Gilbert, Acta Biomater., 2010, 6, 2970-2978.

39 S. Shah, P. T. Yin, T. M. Uehara, S.-T. D. Chueng, L. Yang and K.-B. Lee, Adv. Mater., 2014, 26, 3673-3680.

40 G.-Y. Chen, D. W.-P. Pang, S.-M. Hwang, H.-Y. Tuan and Y.-C. Hu, Biomaterials, 2012, 33, 418-427.

41 H. Kim, J. I. Jang, H. H. Kim, G.-W. Lee, J. A. Lim, J. T. Han and K. Cho, ACS Appl. Mater. Interfaces, 2016, 8, 3193-3199.

42 A. Orza, O. Soritau, L. Olenic, M. Diudea, A. Florea, D. Rus Ciuca, C. Mihu, D. Casciano and A. S. Biris, ACS Nano, 2011, 5, 4490-4503.

43 P. Baei, S. Jalili-Firoozinezhad, S. Rajabi-Zeleti, M. TafazzoliShadpour, H. Baharvand and N. Aghdami, Mater. Sci. Eng., C, 2016, 63, 131-141.

44 M. Kalbacova, A. Broz, J. Kong and M. Kalbac, Carbon, 2010, 48, 4323-4329.

45 J. Y. Lee, Polym. Rev., 2013, 53, 443-459.

46 A. Papadimitropoulos, E. Piccinini, S. Brachat, A. Braccini, D. Wendt, A. Barbero, C. Jacobi and I. Martin, PLoS One, 2014, 9, e102359. 